\title{
Computer assisted customized footwear and traditional micro-cellular rubber (MCR) footwear to reduce recurrence of ulcer for patients with loss of sensation due to leprosy
}

\author{
Karthikeyan Govindasamy ${ }^{\mathrm{a}}$, N. R. Raja ${ }^{\mathrm{b}}$, Pankaj Gupta ${ }^{\mathrm{c}}$, \\ Gaurav Anand ${ }^{\mathrm{d}}$, Premal Das ${ }^{\mathrm{e}}$, Famkima Darlong $^{\mathrm{a}} \&$ \\ Joydeepa Darlong ${ }^{\mathrm{a}}$ \\ ${ }^{\mathrm{a}}$ The Leprosy Mission Trust India, New Delhi, India \\ ${ }^{\mathrm{b}}$ TLM Richardson Leprosy Hospital, Miraj, Maharashtra, India \\ ${ }^{\mathrm{c}}$ TLM Shahdara Hospital, Nand Nagri, New Delhi, India \\ ${ }^{\mathrm{d}}$ TLM Premananda Memorial Leprosy Hospital, Kolkata, West Bengal, India \\ e TLM Naini Hospital, Prayagraj, Uttar Pradesh, India
}

Submitted 20 October 2020; Accepted 30 October 2020

\begin{abstract}
Summary
Objectives: To compare the effectiveness of customized protective footwear made using computer assisted designing/manufacturing (CAD/CAM) technology and current standard micro-cellular rubber (MCR) footwear in the prevention of ulcers.

Methods: A quasi-experimental study was carried at two hospitals of The Leprosy Mission Trust India, where patients with loss of sensation in the foot due to leprosy were included in the intervention (customized insole using CAD/CAM technology) or control (current standard MCR footwear with orthosis) groups. Data were collected using a structured questionnaire at baseline and at 6 and 12 months. The primary outcome was the incidence of ulcer.

Results: A total of 178 patients were included in the final analysis, 81 in the intervention and 97 in the control groups. At the end of follow-up, 10 (12.3\%) patients in the intervention group and $25(25.8 \%)$ patients in the control group developed an ulcer (relative risk, 0.48, [95\% CI, 0.24-0.93], $p$-value $<0.05$ ). The patients' perceived rating of compliance to footwear, comfort, usefulness, participation, and activity level were higher in the intervention group as compared to the control group.

Conclusion: The insole customized using CAD/CAM technology is more effective in preventing recurrent ulcers of the foot due to leprosy as compared to footwear using MCR, with increased compliance and patient perceived comfort, usefulness, participation, and activity level. The new technology used to customize the footwear is feasible.
\end{abstract}

Correspondence to: Karthikeyan Govindasamy, The Leprosy Mission Trust India, New Delhi, India (e-mail: karthikeyan.g@leprosymission.in) 
Keywords: Anaesthetic foot, leprosy, customized insole, protective footwear, ulcer recurrence, ulcers

\section{Introduction}

Leprosy can cause sensory loss including pain sensation in the feet, which therefore need protection from recurrent ulceration and subsequent collapse of the tarsal bones, which often lead to severe foot deformity. In addition, the presence of impairments such as foot drop, claw toes, intrinsic muscle wasting and loss of sweating increases the risk of ulcer. ${ }^{1}$ The incidence of first ulcer increases the risk of subsequent ulcers. ${ }^{2}$ The association between sensory loss in the foot and abnormal pressure distribution while walking has been well established. The sensory loss remains for the lifetime of those with permanent nerve damage and demands continuous care. ${ }^{1,3}$ The leprosy programme has focused on drug distribution and has paid little attention to disability management, as it requires individual attention and treatment plans. A self-care programme and protective footwear using MCR and other soft insoles has been the key strategies used in the prevention of plantar ulcers. ${ }^{4,5}$

In the late 1950s, both Ross and Price described the importance of protective footwear and recommended a hard clog outer sole with a soft sponge rubber insole to protect the foot from developing ulcers due to direct injury and friction. ${ }^{6,7}$ In the early $1960 \mathrm{~s}$, Ward described the effectiveness of MCR footwear in preventing ulcers. ${ }^{8}$ Since then, MCR footwear has been the choice of footwear in leprosy. ${ }^{5}$ The ideal footwear described in the ILEP manual on prevention of disability is in principle the same as described by earlier authors. The footwear should have a soft insole, harder outer sole and a back strap. The soft insole helps to distribute pressure (weight of the body) over the entire plantar surface and protects the vulnerable sites such as the metatarsal heads and heel. The hard outer sole protects from penetration of sharp objects coming into contact with the foot such as thorns and nails. The back strap helps to retain the foot within the open shoe/sandal, as the patient may not realize when the footwear slips away while walking. ${ }^{9}$ Adjustable straps are used to accommodate oedema, deformity, varying size of the foot and/or bandages.

Birke et al., in the early 1990s, investigated the changes in peak pressure with different types of protective footwear used in leprosy. Peak pressure was reduced considerably when using footwear with a $10 \mathrm{~mm}$ thick, soft insole, compared to footwear with harder or thinner insoles. Footwear with a rocker-bottom modification and customized (moulded) insole were also found to significantly reduce the peak pressure when compared to footwear without these modifications. ${ }^{10}$ Their findings emphasize the importance of customization of the insole to improve the fit and thereby its effectiveness. Cross demonstrated the value of podiatry assessment and the importance of customization of MCR footwear by incorporation of foot orthoses in the prevention and management of plantar ulcers, with some success. ${ }^{11}$ However, MCR footwear has been linked with the diagnosis of leprosy and related stigma, so users were often not adhering to the footwear prescription. ${ }^{12,13}$ The manual footwear making with partial orthotic customization is a diminishing art with the dwindling number of skilled shoemakers. As a result, footwears are now mass produced and handed to patients based simply on size. According to the NLEP 2016-17 national report, over 75,000 footwears were provided to people with leprosy, without any customization. ${ }^{14}$ To overcome these barriers, The Leprosy Mission Trust India (TLMTI) tested the feasibility of computer assisted designing/manufacturing (CAD/CAM) technology to fabricate the insole with improved customization for patients with anaesthetic feet due to leprosy. In this report, we present the effectiveness of such 
a

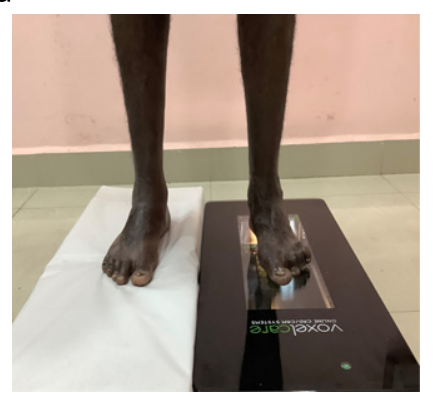

Foot scanning b

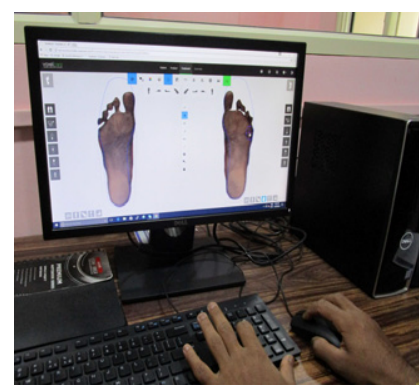

Designing of insole

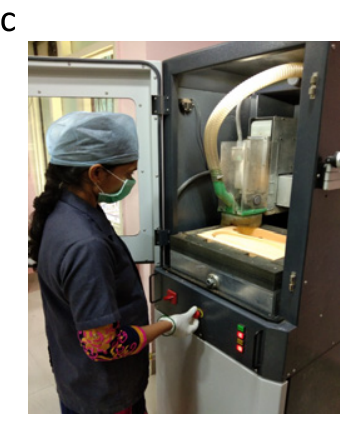

Milling of insole

Figure 1 a to c: Process of foot scanning, designing of insole and milling of the insole from an EVA block.

footwear, henceforth referred to as customized footwear, in the prevention of ulcer recurrence as compared to current standard MCR footwear.

\section{Methodology}

The study was conducted in two referral hospitals for leprosy in central and northern India, of The Leprosy Mission Trust India (TLMTI). One centre was for the intervention group and the other for the control group. In the intervention group, patients who met the inclusion criteria were provided with customized footwear with insoles made using CAD/CAM technology. The insole was fitted into the extra depth footwear and provided to patients. In the control group current standard MCR footwear was provided. In both groups, patients were assessed using the standard podiatry assessment followed in TLMTI and foot orthoses were prescribed and fitted in the footwear. In the intervention group the orthoses were incorporated within the insole, whereas in the control group the orthoses were fitted on top of the MCR insole, as followed in current routine practice. All participants in both groups received self-care instructions to protect their feet, according to the Prevention of Disability protocol of TLMTI, to reduce the variation in the treatment. The self-care instructions were given in the local language. The study was conducted during the period from November 2017 to March 2019.

The study population consisted of patients above 17 years of age, with loss of sensation in the foot due to leprosy, attending the selected study centres. Consecutive patients with insensitive feet, as confirmed by the $10 \mathrm{gm}$ Semmes-Weinstein monofilament, who had experienced an ulcer previously, were included in the study. Patients with other orthopaedic problems, foot drop or corrected foot drop and with amputation (forefoot and above) in a contralateral limb were excluded from the study.

\section{COMPUTER ASSISTED DESIGNING/MANUFACTURING OF INSOLES}

The scanning of the foot was done using a 3D Laser Foot Scanner (LFS). Patients were asked to stand on the scanner in an upright position, one foot at a time. The scanner captured the images of the plantar aspect of the foot within 10 seconds (Figure 1a). The customized insole was designed based on the scanned image of the foot using CAD (Computer Aided Designing) software and orthoses were incorporated as per the prescription of a clinician (Figure 1b). The customized insole was produced in the CAM machine, which carved the insole from an Ethylene Vinyl Acetate (EVA) block, according to the output of the design 
software (Figure 1c). The carved insole was covered on the upper surface with a soft PORON layer (Polyurethane) to reduce the friction between the insole and the foot by increasing the cushioning effect. The insole was then fixed into the extra depth footwear and provided to the patient after minimal adjustments to improve the fit.

The primary outcome of the study was the incidence of ulcer. In this study, an ulcer was considered new if it occurred in a new site, and recurrent if it occurred at the site of a previous ulcer. The secondary outcome measures were the incidence of pre-ulcerative conditions (blister, callus, redness, and cracks), hot foot (Charcot joint), and the patient perceived score of compliance, usefulness, comfort, participation, and activity level.

A standardized structured data collection proforma was designed to collect data on demographic and clinical details at baseline and during follow-up. Patients were assessed at baseline, 6 and 12 months or whenever an ulcer developed. During follow-up, the foot was assessed for the presence of an ulcer, pre-ulcerative conditions and hot foot. The data were collected by the treating therapist. At the final follow-up, patients were asked to rate the footwear using the Visual Analogue Scale, between 0 to 10 on their compliance in using the footwear, perceived usefulness, comfort, participation, and activity level.

The statistical analysis was performed in SPSS version 24. The Chi-square test was used to test the difference in percentage of patients developing an ulcer between the groups. The incidence of ulcer was reported as the number of patients developing an ulcer per 100 personyears at risk (PYAR). The denominator in the incidence rate was obtained by the sum of each patient's length of follow-up, from the date of issue of footwear to the last date of follow-up or the date when the patient developed an outcome, whichever was earlier. The numerator, the total number of patients developing an ulcer, was used to calculate the incidence rate. The perceived comfort, adherence, usefulness, participation and activity level were categorized as poor $(<5)$, acceptable (5-7) or good ( 8 and above) and compared between the groups. In all tests, a $p$-value of $<0.05$ was considered statistically significant.

The study was approved by The Leprosy Mission Trust India Research and Ethics Committee. Information sheets in the local language was provided to all the patients and written informed consent was obtained from all the study participants before inclusion in the study. Participation in the study was voluntary.

\section{Results}

A total of 255 consecutive patients were screened for eligibility to be included in the study. The flow of study participants throughout the period of study is illustrated in Figure 2. In the final analysis, 81 and 97 patients were included, in the intervention and control groups, respectively.

The baseline characteristics of study participants are given in Table 1.

The baseline characteristics of participants was comparable between the groups, except in the foot disability score, overall EHF score and place of residence. Participants in the intervention group had relatively higher foot disability scores and EHF scores, as compared to the control group. A total of $76 \%$ of study participants in the intervention group were from rural areas, as compared to $55 \%$ in the control group, as shown in Table 1.

In the intervention group, 10/81 (12.3\%) patients developed ulcers (total ulcers, both new and recurrent), whereas in the control group 25/97 (25.8\%) patients developed ulcers ( $p$ $<0.05)$. Ulcers at a new site occurred in $5 / 81(6.2 \%)$ of the intervention group and in 8/97 $(8.2 \%)$ of the control group, $(p>0.05)$. Recurrent ulcers occurred in $5 / 81(6.2 \%)$ of the intervention group and 17/97 (17.5\%) of the control group, $(p<0.05)$. The incidence rate of ulcers is shown in Figure 3. The overall incidence of ulcers (both new and recurrent) was 


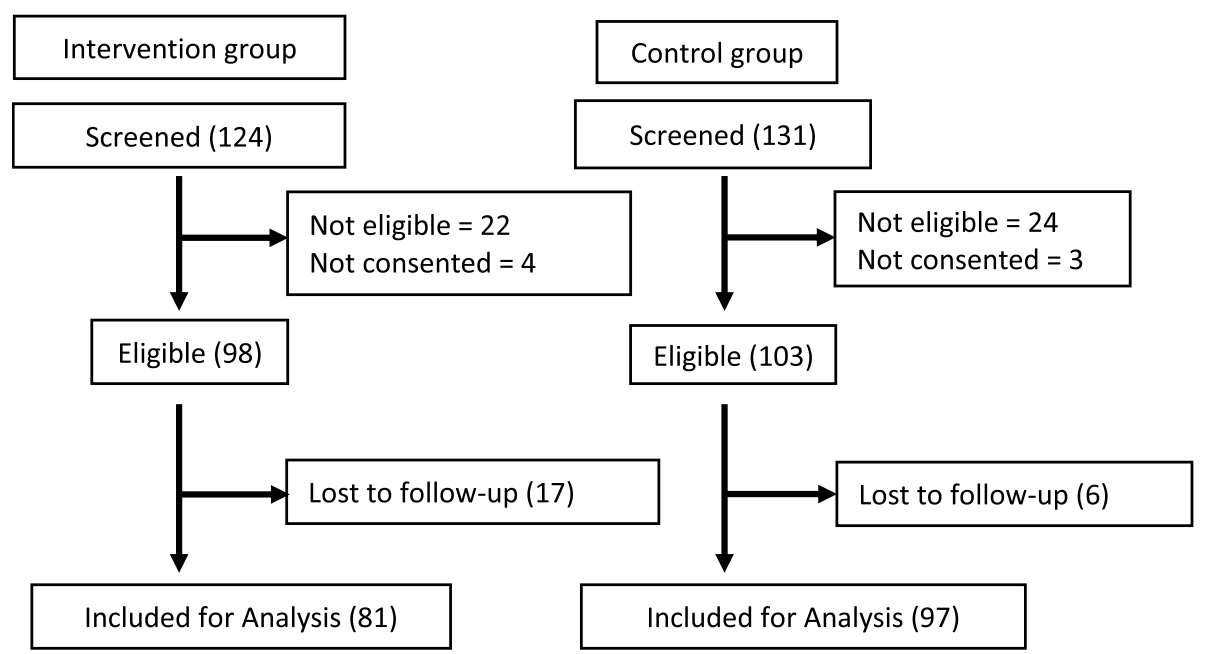

Figure 2 Flow of study participants in the study.

Table 1. Baseline characteristics of study participants in the intervention and control groups

\begin{tabular}{lccc}
\hline Study participants characteristics & $\begin{array}{c}\text { Intervention group } \\
(n=81)\end{array}$ & $\begin{array}{c}\text { Control group } \\
(n=97)\end{array}$ & $p$-value \\
\hline Mean Age in years (SD) & $43(17)$ & $41(16)$ & $>0.05$ \\
Male & $65(80 \%)$ & $73(75 \%)$ & $>0.05$ \\
Female & $16(20 \%)$ & $24(25 \%)$ & $>0.05$ \\
Mean duration of Impairment in months (SD) & $89(70)$ & $70(69)$ & $<0.05$ \\
Foot disability score 1-2* & $37(46 \%)$ & $69(71 \%)$ & $<0.05$ \\
Foot disability score 3-4 & $44(54 \%)$ & $28(29 \%)$ & $<0.05$ \\
Median EHF score (range) & $5(1-10)$ & $3(1-11)$ & $54(55 \%)$ \\
Rural & $64(76 \%)$ & $45(45 \%)$ & $<$ \\
Urban & $20(24 \%)$ & &
\end{tabular}

${ }^{*}$ Foot section of the Eye, Hand, and Foot score.

22 and 52 per 100 PYAR in the intervention and control groups, respectively. The incidence of ulcers at a new site was 11 and 16 per 100 PYAR, and for recurrent ulcers 11 and 35 per 100 PYAR, in the intervention and control groups, respectively.

The relative risk (RR) for developing ulcers and pre-ulcerative signs are shown in Table 2. The RR for developing an ulcer (both new and recurrent) was 0.48 (95\% CIs, 0.24-0.93, $p$ value $<0.05)$ in the intervention group. The RR for developing an ulcer at a new site was 0.75 (95\% CIs, 0.25-2.2, $p$-value $>0.05$ ), and for a recurrent ulcer it was 0.35 (95\% CIs, 0.14$0.91, p$-value $<0.05)$, in the intervention group. The pre-ulcerative signs were observed more frequently in the intervention group 9/81 (11\%) compared to the control group 4/97 (4\%), the difference is not statistically significant $(p$-value $>0.05)$. The relative risk of developing pre-ulcerative signs was 2.7 (95\% CIs, 0.86-8.4, $p$-value $>0.05$ ) in the intervention group.

Study participants in the intervention group consistently rated highly on patient perceived compliance in the use of footwear (92\% vs $63 \%)$, comfort ( $87 \%$ vs $50 \%)$, usefulness (89\% 


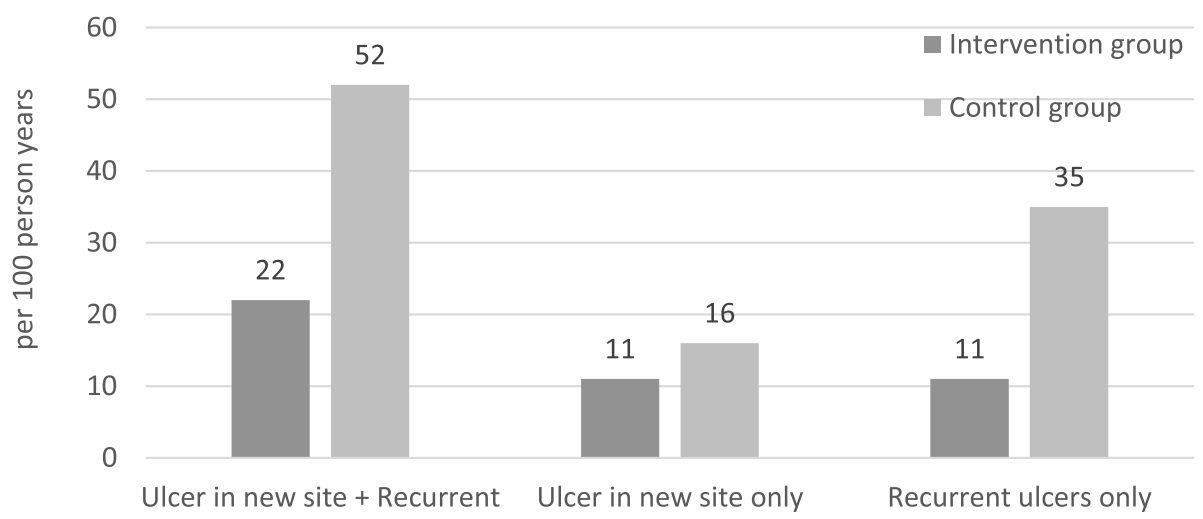

Figure 3 Incidence rate of ulcers in the intervention and control groups (per 100 PYAR).

Table 2. Risk of development of ulcers and pre-ulcerative signs in the intervention and control groups

\begin{tabular}{lccl}
\hline Outcome & $\begin{array}{c}\text { Intervention group } \\
(n=81)\end{array}$ & $\begin{array}{c}\text { Control group } \\
(n=97)\end{array}$ & Relative risk (95\%, CIs) \\
\hline Ulcer in new site + recurrent ulcer & $10(12.3 \%)$ & $25(25.8 \%)$ & $0.48(0.24-0.93)$ \\
Ulcer in new site only & $5(6.2 \%)$ & $8(8.2 \%)$ & $0.75(0.25-2.2)$ \\
Recurrent ulcer only & $5(6.2 \%)$ & $17(17.5 \%)$ & $0.35(0.14-0.91)$ \\
Pre-ulcerative signs & $9(11 \%)$ & $4(4 \%)$ & $2.7(0.86-8.4)$ \\
\hline
\end{tabular}

vs 54\%), participation (95\% vs 58\%) and activity level (93\% vs 63\%) as compared with the control group, as shown in Figure 4. None of the patients in either group developed Charcot foot.

\section{Discussion}

This study aimed to test the computer assisted designing/manufacturing technology in making insoles to improve the customization and acceptability of footwear, thereby reducing recurrence of ulcers. The accurate scanning of the foot and the in-built foot orthotics within the insole improves the customization, and thereby the fit, protecting the vulnerable sites from developing ulcers. This seems to be true as the incidence of ulcers was halved (RR, 0.48, [95\% CIs], 0.24-0.93) among those patients who received the customized footwear as compared with those who used standard MCR footwear. The protection observed was greater for recurrent ulcers $(6.2 \%$ vs $17.2 \%)$ than for new ulcers $(6.2 \%$ vs $8.2 \%)$. A similar effect is observed in diabetic patients at risk of developing foot ulcers, who were provided with customized protective footwear. ${ }^{15,16}$ This is possibly due to two factors. First, because we were able to identify vulnerable sites either through ulcer history or by observing visible scars, appropriate modifications could be made at the corresponding sites in the insole. The modifications were either 'scooping' (removal of part of the insole) so that it completely offloads the site, or through the use of orthoses to reduce the peak pressure at these vulnerable sites. Second, the EVA insole (30-40 shore) is slightly more rigid than the traditional MCR (15 shore), which helps to reduce the movement of metatarsophalangeal joints, thereby decreasing the shearing stress to the tissues under these joints. ${ }^{8}$ However, the low protection against 


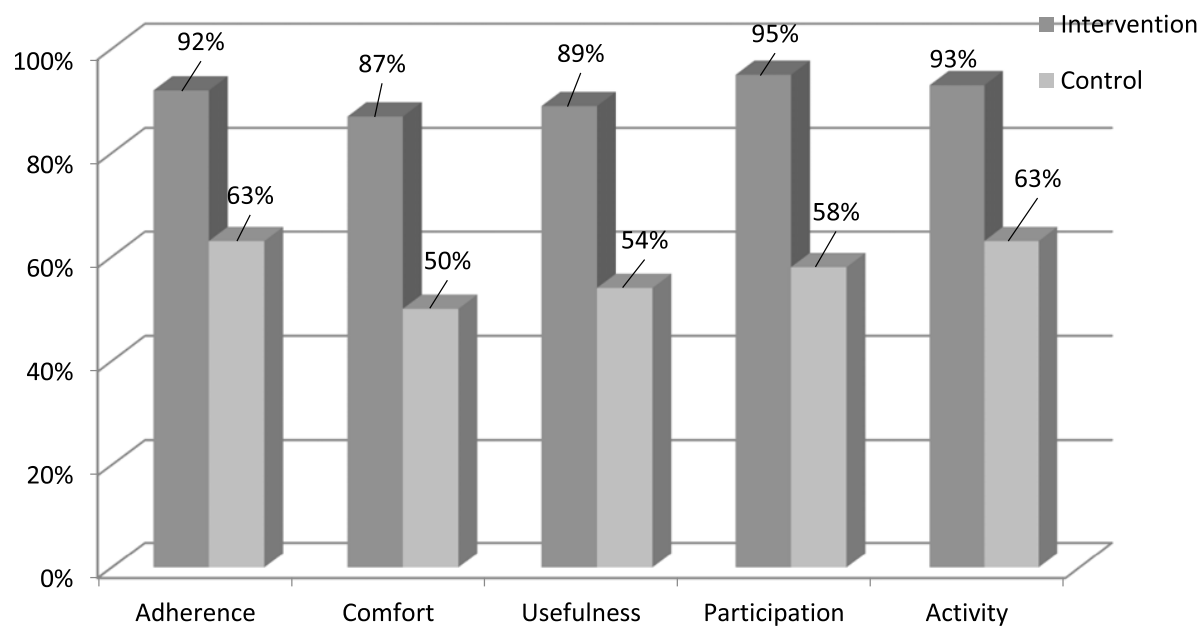

Figure 4 Patient perceived adherence, comfort, usefulness, participation, and activity level after provision of footwear among study participants.

the development of ulcers at new sites needs further investigation with an adequate sample size. The International Working Group on Diabetic Foot care (IWGDF) recommends that the prevention of foot ulcers is effective when the protective footwear has demonstrated a plantar pressure relieving effect while walking. ${ }^{17}$ However, in this study, the change in the plantar pressure before and after footwear provision was not measured to demonstrate the pressure relieving effect.

The incidence of pre-ulcerative conditions such as warmth, redness, cracks and callus in the intervention group was higher than in the MCR group, although the difference was not statistically significant. A similar observation is seen in diabetic feet with protective footwear. ${ }^{18}$ A possible explanation is that some locations on the foot that would ulcerate in the control group might present as pre-ulcerative conditions in the intervention group. Perhaps the improved customization and fit of the insoles prolongs the pre-ulcerative state, or prevents it ulcerating. Therefore, early identification of such conditions and resting of the foot would potentially prevent worsening of the ulcerative condition.

Compliance with the footwear prescription has a direct bearing on the recurrence of an ulcer. ${ }^{19}$ To improve compliance, the footwear designs provided were on par with the designs available in the market, thereby promoting the continuous usage of footwear. In this study, the footwear is considered acceptable by users when the patient adheres to wearing the footwear prescription. The adherence was measured using Visual Analogue scale, on a scale of 0 to 10. The patient perceived rating of adherence was higher in the intervention group as compared to the MCR group. There was a corresponding increase in the patient perceived participation and activity level.

The advantage of the technology tested is that the scanning of the foot can be done at any peripheral location and the images, along with foot assessment details, are stored in closed cloud-based software. This can be retrieved at a central location where the designing and milling of the insole is done. Then the insole is shipped to the periphery and fitted into footwear and provided to the patient. It is best if the designing of the insole is done where the foot scan was taken. 
The cost per pair of shoes with a customized insole is higher than the standard MCR footwear. The benefit of this footwear would outweigh its cost, considering its effectiveness in preventing the recurrence of ulcers. The cost could be considerably reduced by increasing the production or by extending the service to related foot conditions like the diabetic foot. Furthermore, there has been a considerable change in the availability and procurement of materials for insoles within the country which will reduce the cost significantly. A cost-benefit analysis should be considered in future studies.

This report is not without limitations. Firstly, the two different settings for the intervention and control groups was not ideal. However, although different health staff managed the patients in each group, they followed TLMTI guidelines and protocols. There could have been a cultural difference amongst participants in the use of footwear and in the value attached to it. Considering the place of residence of study participants, over 50\% in both groups were from rural areas, so the differences would be minimal. Secondly, the loss to follow-up was $17 \%$ and $6 \%$ in the intervention and control groups, respectively. The major reason for loss to follow-up was migration. Although we were able to contact some of them through telephone to know about their foot and the presence of an ulcer, their data were not included in the analysis because of the lack of verification against the physical examination of the foot. Thirdly, in this study the adherence to footwear was measured using the visual analogue scale, a subjective method of evaluation which can be challenged. However, subjective evaluation of adherence to footwear is found to be acceptable and is used in diabetic foot care as well, in the Indian context. ${ }^{20}$ The variance in compliance with the footwear prescription was not studied objectively to correlate with the outcome, although the higher rating of patient perceived adherence with the customized footwear indicates compliance. Another limitation was that due to resource constraints the outcome was measured by a treating therapist instead of an independent evaluator. In future studies, photographs can be taken and assessed by an independent person blinded to the intervention to evaluate the outcome. ${ }^{21}$ This new footwear with a customized insole is to be studied in a randomized control trial taking into consideration the limitations of the present study in different cultural settings to generate further evidence.

\section{Conclusion}

Customized footwear using computer assisted designing and manufacturing of the insole to improve the fit, is more effective in preventing recurrence of ulcers as compared to current standard MCR footwear and shows improved compliance in usage. The footwear with a customized insole improves patient perceived comfort, usefulness, participation, and activity level.

\section{Acknowledgements}

We acknowledge the financial support received from Google India and Mr R Claushuis towards this footwear project. This study was part of this footwear project. We also acknowledge Dr Jerry Joshua, former Head of Health Programme, Dr Shyamala Anand, former Head of Learning \& Development, Mr Bino S Berry, former Coordinator of Prevention and management of disability of The Leprosy Mission Trust India and other staff who worked on the initial implementation of this project. We sincerely acknowledge all the study participants for their cooperation in completion of this study. 


\section{Ethics approval}

The study was approved by The Leprosy Mission Trust India Ethics Committee. The record number of the approval letter is 5/16/ii, dated 25-11-2016.

\section{Conflict of interest}

Authors declare no conflict of interest.

\section{Funding}

This study was part of the footwear project funded by Google India.

\section{Contributorship}

KG; conceptualized, designed the study methods, data analysis and interpretation, preparation, revision and final approval of the manuscript. RNR; involved in designing of insole, milling and preparation of the insole, supervision of data collection and revision of manuscript. PG \& GA was involved in data collection and revision of the manuscript. PD was involved in approval of the clinical protocol of the intervention, interpretation of analysis and revision and approval of manuscript. FD and JD involved in study design, interpretation of data, revision of manuscript. KG and JD are guarantor.

\section{Patient consent statement}

Informed consent was obtained for all the study participants.

\section{References}

1 Srinivasan H. Prevention of disabilities in patients with leprosy: a practical guide. Geneva: World Health Organization, 1993.

2 Brand P. ILEP. Insensitive feet: a practical handbook on foot problems in leprosy. Brentford: The Leprosy Mission, 1996; p. 88.

3 Watson J. Essential action to minimise disability in leprosy patients. London: TLMI, 1991; p. 36.

4 Chakraborty A, Mahato M, Rao PSSS. Self-care programme to prevent leprosy-related problems in a leprosy colony in Champa, Chattisgarh. Indian J Lepr, 2006; 78(4): 3-11.

5 Karat S. Preventive rehabilitation in leprosy. Lepr Rev, 1968; 39(1): 39-44.

6 Ross W. Footwear and the prevention of ulcers in leprosy. Lepr Rev, 1962; 33: 202-206.

7 Price PE. Studies on plantar ulceration in leprosy. 6. The management of plantar ulcers. Lepr Rev, 1960; 31: 159-171.

8 Ward D. Footwear in leprosy. Lepr Rev, 1962; 33: 94-105.

9 Cross H, Mahato M. ILEP Learning Guide four: How to prevent disability in leprosy. ILEP Learning Guide. London: International Federation of Anti-Leprosy Associations, 2006; p. 64.

10 Birke JA, Foto JG, Deepak S, Watson J. Measurement of pressure walking in footwear used in leprosy. Lepr Rev, 1994; 65(3): 262-271.

11 Cross HA, Sane S, Dey A, Kulkarni VN. The efficacy of podiatric orthoses as an adjunct to the treatment of plantar ulceration in leprosy. Lepr Rev, 1995; 66(0305-7518): 144-157.

12 Lal V, Sarkar D, Das S, Mahato M, Srinivas G. A study to assess the usage of MCR footwear in West Bengal, India. Lepr Rev, 2015; 86(3): 273-277.

13 Gupta P, Karthikeyan G, Nathan RJ. Footwear for the person with an anesthetic foot: what options are available? Lepr Rev, 2017; 88(2): 265-269.

14 National Leprosy Eradication Program. Annual Report 2016-17, Central Leprosy Division Directorate General of Health Services Ministry of Health and Family Welfare Government of India Nirman Bhawan, New Delhi 110011.

15 Bus SA, Waaijman R, Arts M, de Haart M, Busch-Westbroek T, van Baal J et al. Effect of custom-made footwear on foot ulcer recurrence in diabetes: a multicenter randomized controlled trial. Diabetes Care, 2013; 36(12): 4109-4116. 
16 Cavanagh PR, Bus SA. Off-loading the diabetic foot for ulcer prevention and healing. J Vasc Surg, 2010; 52(3): 37s-43s.

17 Bus SA, Armstrong DG, van Deursen RW, Lewis JEA, Caravaggi CF, Cavanagh PR. IWGDF guidance on footwear and offloading interventions to prevent and heal foot ulcers in patients with diabetes. Diabetes Metab Res Rev, 2016; 32: 25-36.

18 Ulbrecht JS, Hurley T, Mauger DT, Cavanagh PR. Prevention of recurrent foot ulcers with plantar pressurebased in-shoe orthoses: the CareFUL prevention multicenter randomized controlled trial. Diabetes Care, 2014; 37(7): 1982-1989.

19 Waaijman R, Keukenkamp R, De Haart M, Polomski WP, Nollet F, Bus SA. Adherence to wearing prescription custom-made footwear in patients with diabetes at high risk for plantar foot ulceration. Diabetes Care, 2013; 36(6): 1613-1618.

20 Viswanathan V, Madhavan S, Rajasekar S, Chamukuttan S, Ambady R. Amputation prevention initiative in South India: positive impact of foot care education. Diabetes Care, 2005; 28(5): 1019-1021.

21 Bowen AC, Burns K, Tong SYC, Andrews RM, Liddle R, O’Meara IM et al. Standardising and assessing digital images for use in clinical trials: a practical, reproducible method that blinds the assessor to treatment allocation. PLoS One, 2014; 9(11): 1-10. 